\title{
Percepções de Pesquisadores sobre Atividades Cooperativas de P\&D: uma Análise com Base na Teoria Institucional
}

\author{
Afrânio Carvalho Aguiar
}

\section{RESUMO}

Estudo visando captar as percepções de pesquisadores de Minas Gerais, experientes em pesquisa cooperativa, quanto a: i) motivação para parcerias em P\&D; ii) dinâmica e estabilidade de alianças; iii) consecução de objetivos fixados para as atividades compartilhadas. Foram analisadas 47 questões, definidas a partir de literatura sobre redes organizacionais e sobre redes cooperativas de pesquisa, respondidas por 305 pesquisadores, de um público-alvo de 673 cientistas. Aplicou-se o método de Analise Fatorial às questões, que então gerou 11 fatores, que se agruparam nas dimensões Criação de Redes, Operação e Resultados. A aplicação da Teoria Institucional revelou alto isomorfismo na percepção dos pesquisadores quanto aos conteúdos das questões, fato revelado pelas técnicas estatísticas empregadas. Foram identificadas pressões coercitivas exercidas pelas Agências de Fomento à Pesquisa, que parecem cultivar mitos racionalizados relativos à eficiência das atividades cooperativas. Aspectos diversos levantados na investigação indicam que o mecanismo de redes cooperativas, para o desenvolvimento das atividades de P\&D, se encontra, no Brasil, em fase de pré-institucionalização.

Palavras-chave: redes organizacionais; redes cooperativas de pesquisa; teoria institucional.

\section{Abstract}

A set of 79 cooperative R\&D projects developed in universities and research centers in Minas Gerais - Brazil were analyzed in order to capture perceptions of 305 researchers, out of 673 experienced scientists on cooperative works, on factors that motivate the establishment of partnerships; stimulate or inhibit the stability of $R \& D$ alliances; facilitate or damage the possibility of arriving at the aimed results. The main theoretical perspective adopted was the Institutional Theory. Data were treated through multivariate statistical analysis, then revealing strong isomorphic behavior of the researchers' perceptions in relation to all the 11 factors generated through the Factorial Analysis technique. This is believed to be due to coercion forces originated from the action of the funding governmental agencies that favor cooperative researches. As no well-structured evaluation system of the performed research activities actually exists in Brazil for allowing justifying partnerships in terms of their effectiveness and efficiency, the stimuli to alliances seem to originate from rationalized myths of the funding agencies. It was found that the process of cooperative research is still in a pre-institutionalized phase among the organizations studied.

Key words: organizational networks; cooperative R\&D networks; institutional theory. 


\section{INTRODUÇÃO}

Principalmente nas duas últimas décadas, nos países desenvolvidos tem-se registrado significativa ampliação das atividades de pesquisa científica e tecnológica em bases cooperativas, por meio da agregação de competências e de recursos materiais das instituições parceiras. Isto ocorre não apenas no caso de alianças entre universidades e entre centros de pesquisa, como também por meio de programas de $\mathrm{P} \& \mathrm{D}$ de natureza cooperativa entre empresas (Schibany \& Polt, 2001).

O impulso para o estabelecimento de alianças entre instituições e entre pesquisadores com o objetivo de realização de trabalhos conjuntos de investigação é simultâneo com o vertiginoso desenvolvimento das tecnologias de informação (Assunção, n.d.; Castells, 1999; Schibany \& Polt, 2001). Esta tendência internacional, um pouco mais tardiamente, também se manifesta no Brasil: é crescente a ação dos órgãos de fomento, tanto em nível federal quanto nas FAP estaduais, induzindo associações entre instituições e entre pesquisadores. $\mathrm{O}$ incremento do trabalho cooperativo tem-se evidenciado pelo menos a partir de 1994 (Longo \& Oliveira, 1999), como atestam iniciativas do MCT/CNPq (Programa PRONEX), da FINEP (Programa RECOPE), da FAPESP (Rede de Seqüenciamento do Genoma), e da FAPEMIG ${ }^{(1)}$ (Programa RECOPE-MG), dentre outras.

Do ponto de vista organizacional, a nova realidade, principalmente no Brasil, onde até agora tem sido pouco estudada, comporta inúmeros e complexos ângulos de análise relativos a essa forma especial de redes organizacionais. Isto motivou o empreendimento de uma pesquisa que objetivou analisar características relativas às percepções dos pesquisadores do Estado de Minas Gerais, com comprovada experiência em atividades cooperativas, quanto aos fatores: i) motivadores das parcerias em $\mathrm{P} \& \mathrm{D}$; ii) determinantes da dinâmica das alianças; e iii) influenciadores da consecução de resultados dos trabalhos desenvolvidos em parcerias.

O suporte teórico para a realização do trabalho foi buscado principalmente na Teoria Institucional. 


\section{Aspectos Té́ricos}

\section{Redes Cooperativas de Pesquisa}

Sebastián (1999, p. 309) conceitua redes cooperativas de pesquisa como “... modalidade de redes de cooperação e [que] se definem pela associação de grupos de pesquisa para a realização de trabalhos conjuntos, geralmente através de projetos de Pesquisa e Desenvolvimento".

Vários autores (De Laat, 1999; Weisz \& Roco, 1995), dão ênfase à busca, por organizações engajadas em alianças de $\mathrm{P} \& \mathrm{D}$, de complementaridade tecnológica e redução do ciclo de vida de produtos e tecnologias. Porter (1999), em direção contrária, alega que os projetos decorrentes de associação entre empresas devem concentrar-se em áreas de pesquisa básica e processos, e não em assuntos relacionados com as fontes de vantagem exclusiva de uma empresa. Por outro lado, reconhece que essas alianças podem também facilitar a percepção de sinalização de áreas técnicas emergentes importantes, que poderão vir a estimular pesquisas individuais e independentes. De forma similar, manifestam-se outros autores (Chotangada, 1997; De Laat, 1999; Longo \& Oliveira, 1999; Lütz, 1999; Ouchi \& Bolton, 1988).

Segundo Longo e Weisz (2000), são de dupla natureza as motivações para a adesão a redes cooperativas: razões estratégicas e razões econômicas. Entre as razões estratégicas mencionam: i) compartilhamento de incertezas; ii) redução do tempo para se chegar a resultados comerciais; iii) troca de informações; iv) compartilhamento de competências; v) monitoramento das mudanças no ambiente; vi) percepção de oportunidades. Entre as razões econômicas incluem a minimização de despesas e o compartilhamento de esforços para possibilitar fazer face aos custos crescentes e à maior complexidade das atividades de inovação tecnológica.

Vários autores citam ainda, como fatores motivadores de alianças em P\&D: i) aumento da complexidade dos problemas científicos e tecnológicos, decorrente do avanço do conhecimento, passando a requerer, cada vez mais, abordagens multidisciplinares (Lütz, 1999); ii) limitação quantitativa das equipes de pesquisadores nas universidades e centros de pesquisa no Brasil; iii) elevação crescente dos custos da pesquisa, decorrentes de fatores como o aumento da sofisticação tecnológica dos equipamentos laboratoriais, reforçando a importância do 'grau de complementaridade dos ativos específicos' (Mazzali \& Costa, 1997); iv) aumento das possibilidades de acesso a recursos para financiamento à pesquisa, em função das políticas indutoras de atividades compartilhadas por parte das Agências de fomento (Longo \& Oliveira, 1999; Sebastián, 1999). 
A respeito da dinâmica das redes cooperativas é importante lembrar o seguinte.

a) Os problemas científicos raramente são resolvidos exatamente conforme o plano original adotado. Haverá necessariamente correção de rotas, surgimento de novas necessidades (recursos humanos e laboratoriais) e modificações várias, que poderão provocar a entrada de novos parceiros na rede. Segundo Nohria (1992), “cada vez que você adiciona uma pessoa, isto torna a vida mais difícil. Cada vez que nós incluímos alguém, ficamos mais próximos de perder o controle" ${ }^{\prime(2)}$.

b) A complexidade dos problemas que são subjacentes ao funcionamento das redes cooperativas faz com que raramente o estabelecimento de uma aliança entre organizações vise, a médio e longo prazo, à execução de um único projeto de pesquisa. Idealmente, as redes devem procurar consolidar recursos e possibilitar relacionamentos duradouros, visando a transações futuras.

c) A separação geográfica favorece a emergência de subculturas divergentes e diminui as possibilidades de integração intergrupo (Nohria, 1992).

d) A desistência de permanência na parceria pode decorrer de incertezas ligadas à natureza da iniciativa, que algumas vezes leva a fracassos, por impossibilidade de combinação das competências dos agentes, extrapolação de custos, ocorrência de comportamentos oportunistas e falta de confiança (Ring, 1999).

A questão da gerência das alianças é apontada por autores diversos (Longo \& Oliveira, 1999; Lütz, 1999; Nohria, 1992; Ring, 1999; Weisz \& Roco, 1995) como de importância vital para o sucesso das iniciativas, dadas às dificuldades relacionadas com a construção e consolidação de uma base de confiança entre os parceiros. Além disto, a multivariedade de atores que as integram pode ser causa de conflitos. As redes cooperativas de pesquisa são, nesse aspecto, ainda mais sensíveis, em virtude das idiossincrasias decorrentes da natureza das atividades e da personalidade dos atores. A coordenação de uma rede de pesquisa atua com fluxos intensos de informação, que devem ocorrer antes de se iniciar o fluxo de outros recursos (Casson \& Cox, 1999). Segundo esses autores, o sucesso da coordenação depende de um fluxo de informação inteligível e confiável. Convém lembrar que nem mesmo este cuidado elimina o risco de decisões inadequadas, por causa da racionalidade limitada (Simon, 1978) e dos diferentes esquemas interpretativos dos indivíduos.

Chotangada (1997) chama atenção para a natureza inexoravelmente sensível da inovação, executada em bases cooperativas, pois envolve o acesso a informação restrita, proprietária. Resulta daí uma tensão inevitável entre a lógica das alianças (redes, sub-contratações, parceria etc) e a lógica da inovação. 
Ring (1999) examina, em especial, o processo de formação de redes de cooperação em $\mathrm{P} \& \mathrm{D}$ e assinala o caráter crítico de questões relacionadas com confiança/desconfiança, retenção de informação relevante e adoção de comportamentos oportunistas. A atividade de pesquisa caracteriza-se como atividade altamente dinâmica, em ambiente fortemente incerto, em função do avanço da fronteira do conhecimento, o que faz com que as organizações de pesquisa se apresentem como organizações orgânicas, segundo dispõe a Teoria Contingencial (Burns \& Stalker, 1961).

Quanto à avaliação de atividades de $\mathrm{P} \& \mathrm{D}$, a experiência internacional, conforme evidencia a análise de autores diversos, demonstra a dificuldade de se avaliarem mais profundamente os resultados decorrentes dos esforços de realização de pesquisas científicas e tecnológicas, pelo menos aqueles que extrapolam os indicadores clássicos de produção científica, como artigos publicados e patentes. Revela igualmente a dificuldade de se aferir em que grau tais resultados impactam a sociedade e a economia (Nelson, 1993).

Com efeito, as principais questões ligadas à avaliação das atividades de Ciência e Tecnologia no Brasil são:

- Inexistência de um sistema consistente, do ponto de vista conceitual e metodológico, de aferição de indicadores de resultados científicos, tecnológicos e principalmente econômicos e sociais referentes às atividades na área de Ciência e Tecnologia.

- Dificuldade de se distinguir com precisão que resultados se referem, de forma inequívoca, a um único projeto (determinado conjunto de artigos publicados, por exemplo). Tal dificuldade decorre do fato de que, normalmente, uma linha de pesquisa ou conjunto de projetos desenvolvidos em determinada rede fazem uso, em certo grau, dos vários aportes financeiros e laboratoriais que cada iniciativa, em dado momento, foi capaz de reunir.

. Fragilidade da cultura de avaliação em nossas instituições de ensino e de pesquisa, o que acaba por comprometer o florescimento de ações efetivas de montagem de sistemas de avaliação de resultados. Uma exceção digna de menção é a correspondente ao Sistema de avaliação de resultados e de impacto socioeconômico dos produtos e serviços da EMBRAPA ${ }^{(3)}$, em operação desde 1996, centralizado em Brasília e registrando as informações de todos os projetos e ações de cada um dos 40 Núcleos Regionais da Empresa existentes no Brasil.

- Necessidade de uma metodologia de comparação de resultados de projetos desenvolvidos 'em rede' com projetos executados em situação de 'não-rede'. 
Os vários critérios e modelos de avaliação existentes possibilitariam a formulação de modelos de análise de redes e projetos de pesquisa, até mesmo no que diz respeito à avaliação de resultados das atividades cooperativas de pesquisa. À falta de sistemas estruturados e já estabilizados de aferição desses resultados, a pesquisa realizada considerou o processo de avaliação mediante a percepção aferida por intermédio de um grupo de pesquisadores e não por medições objetivas.

\section{Teoria Institucional}

A Teoria Institucional resulta da convergência de influência de corpos teóricos originários principalmente da ciência política, da sociologia e da economia (Machado-da-Silva \& Gonçalves, 1999, p. 220). A perspectiva institucional ganhou grande impulso, a partir de 1970, com o advento de várias análises sociológicas do comportamento humano, reconhecendo "fenômenos de racionalidade limitada e o caráter político e contextual da ação social, a partir de uma ótica cognitiva"(4). Dela são expoentes Selznick (1948), March e Olsen (1989), Meyer e Rowan $(1991)^{(5)}$, DiMaggio e Powell (1991)(6), Scott (1995). Os temas mais frequientemente tratados pelos os institucionalistas se relacionam com "valores, poder, cooptação, inovação, modernização, formalismo, conformidade, resistência, contestação, adaptação ambiental, intersubjetividade, linguagem, formação de coalizões políticas e mecanismos de estabelecimento da ordem e da mudança social"(7).

A Teoria Institucional foi formulada com base na observação de que as organizações evoluem não apenas impelidas pelas pressões de grupos internos, mas também por causa de valores da sociedade externa. Machado-da-Silva e Gonçalves (1999, p. 222) chamam a atenção ainda para o fato de que foram Meyer e Rowan, no artigo publicado em $1977^{(8)}$, que "propuseram, em primeira mão, a ampliação conceitual da visão de ambientes em ambientes técnicos e institucionais, como facetas da mesma dimensão". Demandas técnicas e econômicas decorrem do ambiente técnico, e demandas sociais e culturais são postas pelo ambiente institucional.

Através das redes cooperativas de pesquisa fluem recursos de diferentes naturezas e fluem relações sociais importantes (De Laat, 1999; Loughlin \& Búrca, 1995; Lütz, 1999; Powell, 1991; Ring, 1999). Estes fluxos decorrem de demandas ambientais; quando predominam demandas de natureza técnica ou econômica, a ambiência, se atendida de forma eficiente, geralmente retribui às organizações, conferindo-lhes maior legitimidade e maior acesso a recursos. Nas redes cooperativas de pesquisa um desempenho mais eficiente equivaleria a dizer que as parcerias tendem a possibilitar que a atividade de pesquisa seja executada de forma mais eficaz ${ }^{(9)}$. 
Já em ambientes dominados por demandas sociais, as organizações adquirem apoio social e legitimidade, com isto aumentando suas chances de sobrevivência em ambientes turbulentos, ao atenderem as demandas institucionais, por inequívocas influências do ambiente, adequando-se a valores, normas, regras e crenças da sociedade. Tornarem-se mais eficazes e mais legitimadas são formas de as organizações reduzirem suas incertezas e dependências.

A conformidade com as demandas sociais faz com que a organização ganhe apoio social e reduza suas incertezas, não pelo fato de torná-la mais eficiente, mas por coadunar-se com as convenções aceitas. No entanto, a tomada de decisão, que apenas superficialmente se conforma com as normas de racionalidade, pode ser um modo eficaz de legitimar escolhas, muitas vezes tomadas em bases predominantemente emocionais. Meyer e Rowan $(1991)^{(10)}$, citados por Hatch (1997, p. 85),

sugerem que, nas organizações, estes argumentos racionalizados tomam a forma de mitos que não podem ser objetivamente testados, mas que são tomados como racionais na base de que todo o mundo os conhece como verdadeiros. Mitos racionalizados são parte do contexto institucional no qual as organizações operam e aos quais elas se adaptam, a fim de manter sua legitimidade social.

No estudo da execução das pesquisas científicas e tecnológicas, em especial nos processos cooperativos, a Teoria Institucional abre campo para discussões e reflexões sobre aspectos como as seguintes.

- Há evidências bastantes de que as atividades de ciência e tecnologia lograram conseguir legitimidade social?

- O mecanismo de redes cooperativas de pesquisa, que as agências de fomento estimulam, se legitima entre os pesquisadores que delas participam e entre as instituições a que os investigadores se vinculam?

- Seriam tais mecanismos propiciadores de maior eficácia para as atividades de pesquisa?

- Ou seriam estes mecanismos mitos racionalizados, constituídos sob o argumento de otimização na alocação de recursos às atividades de pesquisa?

- As Redes Cooperativas já são, ou poderão vir a ser, mecanismos institucionalizados ou trata-se de modismo passageiro?

Estas são algumas questões que, com os fundamentos da Teoria Institucional, empiricamente se procura analisar neste estudo. 
Machado-da-Silva e Gonçalves (1999, p. 220) comentam que, na visão de Tolbert e Zucker, 1999, quando fundamentadas na Teoria Institucional, há predominância de pesquisas de natureza restritiva, na medida em que "tratam as organizações como dadas pelo ambiente, e a institucionalização como um estado qualitativo: dado arranjo estrutural está ou não institucionalizado na organização". Diferentemente, Tolbert e Zucker (1999, pp. 204-205) conceituam três etapas que entendem constituir o processo de institucionalização; a idéia de processo é também de Scott (1995, p. xiii), segundo o qual "a institucionalização é tanto uma condição quanto um processo", que ocorre quando as ações são repetidas, adquirindo significado similar, que é compartilhado por outros. A adoção de uma estrutura em determinada organização, como inovação gerencial, normalmente tende a induzir o entendimento de que, em organizações semelhantes, a mesma estrutura venha a ser adotada. Dizem as autoras (1999, p. 206) que, "no estágio de pré-institucionalização, [...] (várias) organizações poderão adotar a mesma estrutura; mas elas serão em pequeno número, limitado a um conjunto circunscrito de organizações similares, que enfrentam situações semelhantes". O estágio seguinte da evolução em direção à institucionalização é a Objetificação, correspondendo à difusão da estrutura, o que só ocorre se determinado grau de consenso social a respeito da estrutura houver sido conseguido. Este grau de consenso, por sua vez, depende de uma legitimidade cognitiva e normativa conferida pela teorização. Os esforços de teorização devem demonstrar que a mudança organizacional, associada à estrutura, será bem sucedida. Nesta fase, diz-se que as estruturas estão semi-institucionalizadas, que evolutivamente será seguida pela sedimentação, à qual corresponde a continuidade histórica da estrutura.

Mas que é que faz com que certas ações sejam repetidas? Isto acontece porque há regras explícitas ou leis para garantir sua repetição (influências legais e políticas); ou porque os padrões de atividades são apoiados em normas, valores e expectativas (influências culturais); ou ainda por haver o desejo de ser ou parecer ser como outra instituição (influência social). Powell e DiMaggio (1991) identificam e rotulam essas três diferentes pressões institucionais, criando o conceito de isomorfismo organizacional, por eles definido como sendo o processo pelo qual uma unidade de determinada população é forçada a se assemelhar a outras unidades sujeitas às mesmas pressões ambientais. De acordo com esses autores, quando a pressão para o isomorfismo vem de exigências e regulamentações governamentais, então pressões coercitivas institucionais existem; quando as pressões vêm de expectativas culturais, por exemplo via treinamento profissional de membros da organização, então o que há são pressões normativas institucionais. O desejo manifestado por uma organização de se parecer com outras organizações, consideradas bem sucedidas ou experimentadas, constitui o que denominam pressão mimética institucional surgida de uma compreensível necessidade de 
reduzir incertezas por meio da cópia de outras estruturas, práticas e produção de outras organizações. Quando um ambiente se torna organizado devido a demandas sociais, culturais, políticas ou legais, por intermédio de pressões dessa natureza, diz-se que ele está institucionalizado.

No âmbito das redes cooperativas de pesquisa, a Teoria Institucional poderá auxiliar a entender por que, quase simultaneamente, as agências de fomento à pesquisa passam a estimular e, mais do que isto, a convocar por meio de Editais, em que as regras do jogo são definidas, as entidades executoras de pesquisa a se candidatarem a seus financiamentos para execução de atividades de pesquisa em bases cooperativas. A teoria fornece também elementos para análise dos comportamentos aceitáveis e sancionados no trabalho cooperativo, tais como compartilhamento dos resultados, co-autoria e mútua aprendizagem; constituem regras e convenções cuja observância contribui para a consecução de legitimidade.

\section{Metodologia}

\section{Aspectos Gerais}

Procedeu-se a uma pesquisa exploratória para identificar as iniciativas de pesquisa existentes no Estado de Minas Gerais com caráter de provável atividade cooperativa. Uma pesquisa quantitativa descritiva, tipo survey, de característica transversal, teve seqüência, quando então, além de outros elementos, foram identificados os pesquisadores que efetivamente tinham ou tiveram experiência em trabalho de pesquisa cooperativa (dependendo de a pesquisa cooperativa estar em andamento ou, ao contrário, estar concluída). A etapa subsequiente da pesquisa, tipo survey, constituiu-se do levantamento das percepções dos pesquisadores quanto aos fatores motivadores do trabalho cooperativo e aos facilitadores ou inibidores da consecução dos objetivos das ações cooperativas.

O processo de 'garimpagem' resultou na identificação e no mapeamento de 239 iniciativas (redes e projetos), que apenas em parte corresponderam aos limites conceituais pré-definidos na pesquisa, para trabalhos cooperativos; como, por exemplo, o caráter de aliança formal. Este trabalho constituiu a primeira fase da pesquisa. Outra etapa da investigação descreveu estas iniciativas e obteve-se, como resultado, uma relação de 754 pesquisadores participantes de 79 redes e projetos cooperativos identificados, considerados enquadráveis nos limites predeterminados. Desses pesquisadores, alguns atuavam em 2 ou até em 3 redes ou projetos cooperativos de forma que, ao se eliminarem as participações múltiplas, restaram 673 pesquisadores diferentes, que passaram a constituir o público-alvo 
na terceira etapa da pesquisa exploratória explicativa. Obteve-se o questionário respondido de 305 pesquisadores, o que representou uma amostra de 45,32\% do público-alvo.

A definição utilizada para a amostra apresenta simultaneamente características de amostragem intencional e por julgamento (Malhotra, 2001; Sampieri, Collado, \& Lucio, 2003), método em que o pesquisador "pode [...] selecionar a amostra baseado no próprio conhecimento da população e de seus elementos e da natureza das metas da pesquisa" (Malhotra, 2001). Ambos os métodos são processos de amostragem não-probabilística, o que enfraquece a capacidade preditiva das conclusões da pesquisa, comprometendo as generalizações mais amplas dos resultados.

Como mencionado, apenas parcerias formalizadas mediante contratos ou convênios foram consideradas. Não se nega aqui, certamente, a importância dos laços informais na dinâmica das parcerias em Ciência e Tecnologia. A pesquisa empreendida, no entanto, teve clara intenção de estudar aspectos como comprometimento das partes, responsabilidades, deveres, direitos, riscos de oportunismo etc., o que tornou necessário fossem tratadas na investigação apenas as alianças formais. Não foram impostos limites adicionais com relação a horizonte temporal e cobertura temática das iniciativas estudadas.

\section{Levantamento das Percepções dos Pesquisadores: Fatores de Motivação e Fatores Passíveis de Afetar a Consecução dos Objetivos de Ações Cooperativas ( $3^{\text {a Etapa) }}$}

Além de não se conhecer a priori se as 239 iniciativas de cooperação identificadas na pesquisa exploratória atendiam aos demais limites fixados pela pesquisa, dois outros problemas contribuíram para o arrefecimento do desejo de se procurar correlacionar, de forma concreta e mensurável, os resultados efetivos das atividades de pesquisa, com a condição de serem os projetos desenvolvidos dentro de estruturas de redes cooperativas:

a) A atividade de pesquisa, por sua natureza intrínseca, dificilmente consegue vincular, de forma inequívoca, os resultados obtidos com os projetos individualmente considerados. A rigor, projetos diversos correlacionados, financiados por diferentes fontes, materializando uma linha de pesquisa de um pesquisador ou de um grupo de pesquisa é que, ao final de um dado tempo, apresentam um conjunto de resultados aferíveis.

b) As entidades brasileiras executoras de atividades de pesquisa não mantêm serviços muito bem estruturados de controle da produção científica de 
pesquisadores, grupos, departamentos e laboratórios. As informações, quando disponíveis, são dispersas, fragmentadas, registradas em meios de difícil recuperação, como é o caso de relatórios impressos.

Em face dessas dificuldades, optou-se por proceder ao levantamento de expectativas e resultados esperados de projetos executados dentro das estruturas das redes identificadas. Esta etapa da pesquisa compreendeu inicialmente a elaboração de um modelo de análise composto essencialmente de três Sistemas ou Dimensões de análise, composto por construtos e construtos associados, como a seguir se indicam.

- Criação e Implantação de Redes e Projetos Cooperativos, que se transformaram em dois construtos associados: i) Pré-Condições e ii) Motivação

- Operação de Atividades Cooperativas, estudo por meio de seis construtos associados: i) Confiança; ii) Comportamentos; iii) Competição; iv) Liderança; v) Gestão; vi) Financiadores.

- Produção de Resultados (Eficiência), compreendendo três construtos associados: i) Resultados não-científicos; ii) Resultados Operacionais; iii) Resultados Científicos.

Os construtos principais e os construtos associados foram operacionalizados, no conjunto, por meio de 47 itens de um questionário distribuído aos respondentes por meio da Internet, em sistema automatizado de coleta de dados especialmente adaptado $^{(11)}$ para o projeto. As questões que compuseram o questionário foram escolhidas com base em tópicos da literatura sobre Redes Organizacionais, Redes Cooperativas de Pesquisa e Teoria Institucional.

\section{Resultados da Pesquisa Empírica}

\section{As Agências de Financiamento como Indutoras de Ações Cooperativas}

Em 62,05\% dos casos (Tabela 1), as redes e projetos cooperativos identificados surgem como resposta a ações indutoras das agências financiadoras. Uma análise da documentação emitida pela FAPEMIG, sob a forma de editais, mecanismo de convocação de propostas de projeto, pela primeira vez utilizada na Fundação em julho de 1996, revela que, desde a introdução desse mecanismo até outubro de 2004, foram divulgados 51 editais (Tabela 2). Esses editais revelam que a exigência 
de trabalho cooperativo apresenta gradações que poderíamos classificar de INEXISTENTE, MUITO BAIXA, BAIXA, MÉDIA, ALTA e MUITO ALTA e que, inequivocamente, ao longo do tempo, aumenta-se a importância, pelo menos percentualmente, da exigência de 'trabalho cooperativo' expressa nos editais. Indiscutível também é o fato de que, no período, as agências federais aumentaram sua indução de ações cooperativas, como provam os editais do Programa RECOPE - Redes Cooperativas de Pesquisa, da FINEP e do Programa PRONEX do CNPq.

Tabela 1: Número de Redes e Projetos Cooperativos, por Programa do Agente Financiador

\begin{tabular}{|c|c|c|c|c|}
\hline $\begin{array}{c}\text { Agente } \\
\text { financiador }\end{array}$ & Programa & $\begin{array}{l}\mathbf{N}^{0} \text { redes/ } \\
\text { projetos }\end{array}$ & $\begin{array}{l}\text { Freqüiência } \\
\%\end{array}$ & $\begin{array}{c}\text { Frequiência } \\
\text { acumulada \% }\end{array}$ \\
\hline $\begin{array}{l}\text { CNPq, } \\
\text { FINEP e } \\
\text { CAPES }\end{array}$ & $\begin{array}{l}\text { Programa de apoio ao desenvolvimento } \\
\text { cientifico e tecnológico - PADCT }\end{array}$ & 8 & 10,13 & 10,13 \\
\hline CNPq & $\begin{array}{l}\text { Programa de apoio a núcleos de excelência - } \\
\text { PRONEX }\end{array}$ & 8 & 10,13 & 20,26 \\
\hline CNPq & $\begin{array}{c}\text { Programa de Capacitação de R. Humanos para } \\
\text { Atividades Estratégicas - RHAE }\end{array}$ & 2 & 2,53 & 22,79 \\
\hline $\begin{array}{l}\text { EMBRAPA } \\
\text { /Funcafé }\end{array}$ & $\begin{array}{l}\text { Fundo de Defesa da Economia Cafeeira/ } \\
\text { Programa Brasileiro de Pesquisa Cafeeira }\end{array}$ & 1 & 1,27 & 26,06 \\
\hline EMBRAPA & $\begin{array}{c}\text { Rede nacional de ensaios de cultivares de } \\
\text { alfafa - RENACAL }\end{array}$ & 1 & 1,27 & 25,33 \\
\hline $\begin{array}{l}\text { EMBRAPA } \\
\text { / BIRD }\end{array}$ & $\begin{array}{l}\text { Projeto de apoio ao desenvolvimento da tecn. } \\
\text { agropecuária para o Brasil - PRODETAB }\end{array}$ & 3 & 3,80 & 29,13 \\
\hline EMBRAPA & Sistema de produção de agricultura familiar & 1 & 1,27 & 30,04 \\
\hline FAPEMIG & Editais diversos & 4 & 5,06 & 35,46 \\
\hline $\begin{array}{l}\text { FAPEMIG/ } \\
\text { BDMG }\end{array}$ & Desenvolvimento Tecnológico Industrial & 2 & 2,53 & 37,99 \\
\hline FINEP & $\begin{array}{c}\text { Redes cooperativas de pesquisa em } \\
\text { Engenharia - RECOPE }\end{array}$ & 17 & 21,52 & 59,51 \\
\hline FIOCRUZ & $\begin{array}{c}\text { Programa de apoio à pesquisa estratégica em } \\
\text { Saúde - PAPES }\end{array}$ & 1 & 1,27 & 60,78 \\
\hline MCT & $\begin{array}{c}\text { Projeto Genoma Brasileiro } \\
\text { Não-vinculados a Programas (Demanda } \\
\text { Espontânea,Contratos c/Empresa) }\end{array}$ & $\begin{array}{c}1 \\
30\end{array}$ & $\begin{array}{l}1,27 \\
37.97\end{array}$ & $\begin{array}{c}62,05 \\
100,00\end{array}$ \\
\hline
\end{tabular}

Tabela 2: Classificação do Grau de Exigência de Parceria dos Editais da FAPEMIG

\begin{tabular}{|c|c|c|c|c|c|c|c|c|c|}
\hline \multirow{2}{*}{$\begin{array}{c}\text { GRAU DE EXIGÊNCIA DE } \\
\text { PARCERIA } \\
\end{array}$} & \multicolumn{9}{|c|}{ ANOS } \\
\hline & 1996 & 1997 & 1998 & 1999 & 2000 & 2001 & 2002 & 2003 & $2004^{*}$ \\
\hline $\begin{array}{l}\text { INEXISTENTE/ } \\
\text { MUITO BAIXO }\end{array}$ & 2 & 0 & 0 & 0 & 0 & 6 & 4 & 3 & 0 \\
\hline BAIXO/MÉDIO & 0 & 0 & 1 & 0 & 1 & 6 & 8 & 3 & 0 \\
\hline ALTO/MUITO ALTO & 0 & 0 & 0 & 1 & 0 & 5 & 5 & 3 & 3 \\
\hline TOTAL & 2 & 0 & 1 & 1 & 1 & 17 & 17 & 9 & 3 \\
\hline
\end{tabular}

* Até outubro de 2004. 


\section{Caracterização das Redes por Área Temática e dos Respondentes}

As áreas temáticas a que se vinculam as 79 redes e projetos cooperativos identificados foram classificadas segundo a Tabela de Classificação de Áreas de Conhecimento do CNPq.

Analisados os registros de forma mais agregada, constata-se que 23 iniciativas correspondem à área das Engenharias, certamente uma consequiência do Programa Redes Cooperativas de Pesquisa em Engenharia-RECOPE; 19 outras se vinculam à área Agropecuária e 15 à área de Ciências Biológicas. Esses dados guardam coerência com as áreas onde a atividade de pesquisa em Minas Gerais é mais vigorosa, como se constata pelo Diretório dos Grupos de Pesquisa do CNPq, ano 2004 e anteriores: Agronomia e áreas correlatas: 9,33\%; Engenharia Metalúrgica. Metalurgia: 9,33\%; Ciência dos Materiais: 8,00\%; Química. FísicoQuímica: 5,33\%.

Os 305 pesquisadores $\left(6,04 \%\right.$ dos $5.052^{(12)}$ pesquisadores de Minas Gerais) respondentes foram classificados segundo as categorias abaixo relacionadas e os resultados são os que se seguem.

- Titulação Máxima.

Graduação: $\quad 5$ respondentes $(1,6 \%) \quad$ Especialização: $\quad 8$ respondentes $(2,6 \%)$

Mestrado: $\quad 41$ respondentes $(13,4 \%) \quad$ Doutorado: $\quad 251$ respondentes $(82,3 \%)$

. Tempo de Experiência em Atividade de Pesquisa.

Mais de 25 anos: 95 respondentes $(31,1 \%) \quad$ De 5 a 10 anos: $\quad 30$ respondentes $(9,8 \%)$

De 20 a 25 anos : 67 respondentes $(22,0 \%) \quad$ De 2 a 5 anos: 5 respondentes $(1,6 \%)$

De 15 a 20 anos: 49 respondentes $(16,1 \%) \quad$ Até 2 anos: 3 respondentes $(1,0 \%)$

De 10 a 15 anos: 56 respondentes $(18,4 \%)$

- Categoria de Instituição a que se vincula o pesquisador.

Universidade 189 respondentes $(62,0 \%) \quad$ Universidade/Escola 11 respondentes $(13,6 \%)$

Pública: Privada:

Instituto de 50 respondentes $(16,4 \%) \quad$ Empresa Privada: 7 respondentes $(2,3 \%)$

Pesquisa

Federal:

Instituto de 43 respondentes $(13,8 \%)$

Pesquisa

Outros órgãos $\quad 6$ respondentes $(2,0 \%)$

Estadual: governamentais: 
. Categorização por nível de classificação do Pesquisador no CNPq.

$\begin{array}{llll}\text { Nível 1A : } & 22(7,2 \%) & \text { Nível 1C: } & 28(9,2 \%) \\ \text { Nível 2A : } & 33(10,8 \%) & \text { Nível 2C: } & 21(6,9 \%) \\ \text { Nível 1B: } & 22(7,2 \%) & \text { Recém-doutor: } & 8(2,6 \%) \\ \text { Nível 2B: } & 27(8,9 \%) & \begin{array}{l}\text { Não recebeu } \\ \text { classificação: }\end{array} & 144(47,2 \%)\end{array}$

\section{Análise dos Escores Atribuídos, na Escala Likert, aos Itens de Operacionalização dos Construtos}

Os escores atribuídos a cada um dos 47 itens constantes do Questionário foram analisados individualmente, tendo como referência o arcabouço teórico utilizado na pesquisa. Estes resultados não são mostrados aqui, mas subsidiam algumas conclusões mencionadas no item 5 deste trabalho.

\section{Percepções dos Pesquisadores quanto a Fatores que Motivam a Criação e Implantação, Operação e Eficiência das Redes Cooperativas}

Pela dificuldade intrínseca de se fazer uma interpretação coerente, a partir das respostas obtidas individualmente de um conjunto de 47 questões, procedeu-se a uma análise fatorial com a finalidade de se reduzir o número de variáveis, agrupando-as em fatores, cuja variância total fosse capaz de corresponder ao máximo possível da variância correspondente ao conjunto dos itens ou questões.

A Análise Fatorial permitiu a identificação de 11 fatores, como se mostra na Tabela 3, cuja análise da confiabilidade interna foi medida pelo Coeficiente Alfa de Cronbach, resultando em valor $\alpha>0,60 \mathrm{em}$ todos os casos, como apresenta a terceira coluna da Tabela 3. A variância dos 11 fatores corresponde a $65 \%$ da variância total correspondente às 47 questões. 


\section{Tabela 3: Determinação do Número de Fatores Baseada na Análise de Componentes Principais* e Medidas da Confiabilidade Interna, para Cada Fator}

\begin{tabular}{|c|c|c|c|c|c|c|c|}
\hline \multirow[t]{2}{*}{ Fatores } & \multirow{2}{*}{\multicolumn{3}{|c|}{ Fator $/ \mathbf{N}^{0}$ de questões }} & \multirow{2}{*}{$\begin{array}{c}\text { Alfa de } \\
\text { Cronbach }\end{array}$} & \multicolumn{3}{|c|}{ Soma de quadrados de cargas com Rotação } \\
\hline & & & & & Autovalor & \% de variância & $\%$ acumulada \\
\hline I & \multicolumn{3}{|c|}{ Gestão (7 questões) } & 0,86 & 4,78 & 10,9 & 10,9 \\
\hline II & \multirow{2}{*}{\multicolumn{3}{|c|}{$\begin{array}{l}\text { Motivação ( } 7 \text { questões) } \\
\text { Comportamentos (5 questões) }\end{array}$}} & 0,84 & 3,93 & 8,9 & 19,8 \\
\hline III & & & & 0,82 & 3,34 & 7,6 & 27,4 \\
\hline IV & \multicolumn{3}{|c|}{$\begin{array}{l}\text { Resultados operacionais (3 } \\
\text { questões) }\end{array}$} & 0,78 & 2,56 & 5,8 & 33,2 \\
\hline V & \multicolumn{3}{|c|}{ Financiadores (4 questões) } & 0,75 & 2,48 & 5,6 & 38,8 \\
\hline VI & \multicolumn{3}{|c|}{ Liderança (3 questões) } & 0,60 & 2,25 & 5,1 & 43,9 \\
\hline VII & \multicolumn{3}{|c|}{$\begin{array}{l}\text { Resultados científicos (3 } \\
\text { questões) }\end{array}$} & 0,73 & 2,17 & 4,9 & 48,9 \\
\hline VIII & \multicolumn{3}{|c|}{$\begin{array}{l}\text { Resultados não-científicos (4 } \\
\text { questões) }\end{array}$} & 0,63 & 1,88 & 4,3 & 53,1 \\
\hline IX & \multicolumn{3}{|c|}{ Pré-condições (5 questões) } & 0,55 & 1,86 & 4,2 & 57,4 \\
\hline$X$ & \multicolumn{3}{|c|}{ Competição (3 questões) } & 0,77 & 1,85 & 4,2 & 61,6 \\
\hline $\mathrm{XI}$ & \multirow{2}{*}{\multicolumn{3}{|c|}{ Confiança (3 questões) }} & 0,87 & 1,52 & 3,5 & 65,0 \\
\hline Total & & & & & 28,62 & 65,0 & $\underline{-}$ \\
\hline \multicolumn{8}{|c|}{ 'Método de Rotação $\rightarrow$} \\
\hline Legenda: $\mathrm{I}$ & Fatores: & $\begin{array}{l}\mathrm{I} \rightarrow \text { Gestão } \\
\text { IV } \rightarrow \text { Resultad } \\
\text { VII } \rightarrow \text { Resulta } \\
\mathrm{X} \rightarrow \text { Competic }\end{array}$ & $\begin{array}{l}\text { s operacio } \\
\text { os científic } \\
\text { lo }\end{array}$ & $\begin{array}{l}\text { II - } \\
\text { V - } \\
\text { VII } \\
\text { XI }\end{array}$ & $\begin{array}{l}\text { Motivação } \\
\text { Financiadores } \\
\rightarrow \text { Resultados n } \\
\text { Confiança }\end{array}$ & científicos & $\begin{array}{l}\rightarrow \text { Comportamentos } \\
\rightarrow \text { Liderança } \\
\rightarrow \text { Pré-condições }\end{array}$ \\
\hline
\end{tabular}

\section{Análise Descritiva e Comparativa dos Fatores}

Pode-se apresentar as medidas dos 11 fatores gerados pela análise fatorial, considerados os escores atribuídos por todos os respondentes, sem qualquer segmentação da amostra, indicando, para cada um deles, os seus valores mínimo, médio, máximo e desvio-padrão. Verificar-se-á que, observados os níveis de significância $(\mathrm{p}<0,005)$, na percepção dos pesquisadores da amostra há respectivamente maior concordância com as afirmações contidas nas Questões correspondentes (maiores médias para os escores resultantes) aos seguintes fatores, ordenados segundo sua classificação (Tabela 4):

$1^{\circ}$ - Fatores Confiança e Gestão

$2^{\circ}$ - Fatores Pré-Condições, Motivação, Comportamentos, Resultados nãocientíficos e Resultados científicos 
$3^{\circ}$ - Fator Liderança

$4^{\circ}$ - Fator Resultados operacionais

$5^{\circ}$ - Fatores Competição e Órgãos financiadores

Esta representa, pois, a ordem de importância que os respondentes atribuem aos construtos que se relacionam com a motivação, a dinâmica e os resultados das atividades cooperativas. A aderência do fato decorrente dessa categorização dos postulados teóricos assumidos será comentada no item 5. Dado que o sentido das questões era sempre afirmativo (Ex: existência de confiança entre os parceiros afeta favoravelmente a consecução dos objetivos das parcerias, ou o espírito de competição entre as entidades/participantes favorece a consolidação das alianças), os dados que a Tabela 4 demonstram a concordância dos respondentes com o conceito correspondente ao item.

\section{Tabela 4: Medidas Descritivas das Médias dos Fatores do Modelo de} Análise, Considerada a Amostra Total de Respondentes

\begin{tabular}{|c|c|c|c|c|c|}
\hline \multirow[b]{2}{*}{ Fatores } & \multirow[b]{2}{*}{$\mathbf{n}$} & \multicolumn{4}{|c|}{ Medidas descritivas } \\
\hline & & Mínimo & Máximo & Média & d.p. \\
\hline \multicolumn{6}{|l|}{ Criação de Rede } \\
\hline Pré-condições & 301 & 2,7 & 7,0 & 5,6 & 0,9 \\
\hline Motivação & 299 & 1,4 & 7,0 & 6,0 & 0,9 \\
\hline \multicolumn{6}{|l|}{ Operação de Rede } \\
\hline Confiança & 304 & 1,0 & 7,0 & 6,5 & 0,8 \\
\hline Comportamentos & 298 & 2,2 & 7,0 & 5,7 & 1,1 \\
\hline Competição & 303 & 1,0 & 7,0 & 3,7 & 1,6 \\
\hline Liderança & 301 & 1,3 & 7,0 & 5,2 & 1,2 \\
\hline Gestão & 295 & 1,0 & 7,0 & 6,3 & 0,8 \\
\hline Financiadores & 297 & 1,0 & 7,0 & 3,9 & 1,3 \\
\hline \multicolumn{6}{|l|}{ Resultados de Rede } \\
\hline Resultados Não Científicos & 304 & 1,0 & 7,0 & 5,7 & 1,1 \\
\hline Resultados Operacionais & 297 & 1,0 & 7,0 & 4,9 & 1,3 \\
\hline Resultados Científicos & 304 & 1,7 & 7,0 & 5,6 & 1,1 \\
\hline
\end{tabular}

Nota: $\mathrm{p}<0,0001$ (O valor de p refere-se à Análise de Variância de 1 fator baseado num modelo em bloco)

Conclusão: $(\mathrm{E}=\mathrm{H})<\mathrm{J}<\mathrm{F}<(\mathrm{A}=\mathrm{B}=\mathrm{D}=\mathrm{I}=\mathrm{K})<(\mathrm{C}=\mathrm{G})$

Legenda: Fatores:

$\mathrm{A} \rightarrow$ Pré-condições $\quad \mathrm{B} \rightarrow$ Motivação

$\mathrm{C} \rightarrow$ Confiança $\quad \mathrm{D} \rightarrow$ Comportamentos

$\mathrm{E} \rightarrow$ Competição

$\mathrm{G} \rightarrow$ Gestão

$\mathrm{I} \rightarrow$ Resultados não-científicos

$\mathrm{K} \rightarrow$ Resultados científicos

$\mathrm{F} \rightarrow$ Liderança

$\mathrm{H} \rightarrow$ Financiadores

$\mathrm{J} \rightarrow$ Resultados operacionais 


\section{Segmentação da Amostra}

As medidas descritivas das médias dos fatores foram então calculadas para segmentos da amostra determinados em consonância com a caracterização indicada no item Caracterização das redes por área temática e dos respondentes, considerando-se os agrupamentos indicados abaixo:

. Agrupamento a (Grupo I=Doutores e Grupo II = Mestres e Especialistas).

- Agrupamento b (Grupo III = Experiência em Pesquisa até 15 anos e Grupo IV = Experiência em Pesquisa de 15 anos ou mais).

- Agrupamento c (Grupo V = Vínculo com Universidade ou Escola Isolada Pública; Grupo VI = Vínculo com Instituto de Pesquisa Federal; Grupo VII = Vínculo com Instituto de Pesquisa Estadual).

- Agrupamento d (Grupo VIII = Classificação CNPq 1A e 2A; Grupo IX = Classificação CNPq 1B e 2B; Grupo X= Classificação CNPq 1C e 2C; Grupo $\mathrm{XI}=$ Não recebeu classificação do $\mathrm{CNPq}$ ).

Resultaram tabelas de escores por segmentos da amostra, não reproduzidas neste trabalho, que, por comparação com a Tabela 4 e entre agrupamentos, permitem concluir que inexistem diferenças significativas $(\mathrm{p}<0,05)^{(13)}$ entre pesquisadores.

. Dos Grupos I e II, quaisquer que sejam os fatores estudados; portanto, nível de titulação não distingue os pesquisadores quanto à maneira como percebem os fatores pertinentes à análise das redes e projetos cooperativos.

- Dos Grupos III e IV, quaisquer que sejam os fatores estudados, constatando-se o mesmo fato, quando a distinção entre os pesquisadores se faz com relação a seu tempo de experiência em pesquisa.

- Dos quatro grupos de pesquisadores, quanto a qualquer fator, quando o critério de segmentação é o de Classificação CNPq.

Na comparação das medidas referentes aos Grupos V, VI e VII, a análise por segmento da amostra evidenciou a existência de diferenças significativas apenas quanto ao Fator G (Gestão), a que corresponderam sete questões do Questionário, e ao Fator I (Resultados não-científicos), a que correspondem duas questões do Questionário. Com efeito, no que diz respeito à Gestão, não houve diferenças significativas ( $p>0,05)$ entre os Grupos V e VI; mas esses dois grupos tenderam a concordar mais fortemente com as afirmativas referentes às questões correspondentes ao Fator G (Gestão). Pesquisadores vinculados a Universidades/ 
Escolas Públicas Isoladas apresentaram médias significantemente superiores às correspondentes aos Institutos de Pesquisa Estaduais.

Quanto ao Fator I, o Grupo VI foi o que apresentou médias maiores e significativamente superiores $(\mathrm{p}<0,005)$ às dos Grupos V e VII que, entre si, não apresentaram diferenças relevantes $(p>0,05)$ quanto a esse fator.

\section{Análise da Formação de Agrupamentos pelos Elementos Amostrais}

Foi utilizada a análise de conglomerados (Cluster Analysis) para identificar possíveis agrupamentos de pesquisadores da amostra, formados em consonância com suas percepções sobre os 11 fatores gerados pela análise fatorial. A análise indicou apenas dois conglomerados (clusters) distintos: o primeiro foi constituído por 90,9\% dos pesquisadores, aos quais corresponderam os maiores escores médios em todos os fatores avaliados (Grupo dos CONCORDANTES); o segundo foi constituído por $9,1 \%$ dos pesquisadores, aos quais corresponderam os menores escores médios (Grupo dos DISCORDANTES).

Tabela 5: Escores Médios dos Fatores de Interesse em Relação aos Dois Grupos de Respondentes segundo o Grau de Concordância com os Itens

\begin{tabular}{l|c|c}
\hline \hline \multicolumn{1}{c|}{ Fatores } & \multicolumn{2}{c}{ Nível de Concordância } \\
\cline { 2 - 3 } & Alto & Baixo \\
\hline Criação de Rede & & \\
$\quad$ Pré-condições (Criação de Rede) & 5,6 & 4,9 \\
Motivação (Criação de Rede) & 6,1 & 4,3 \\
Operação de Rede & & \\
Confiança & 6,6 & 5,7 \\
Comportamentos & 5,8 & 4,0 \\
Competição & 3,8 & 2,4 \\
Liderança & 5,3 & 3,9 \\
Gestão & 6,4 & 5,4 \\
Financiadores & 4,0 & 3,2 \\
Resultados referentes à Rede & & \\
Resultados Não Científicos & 5,9 & 3,9 \\
Resultados Operacionais & 5,1 & 2,9 \\
Resultados Científicos & 5,8 & 4,0 \\
\hline \hline
\end{tabular}

Nota: Os grupos foram formados a partir de análise de conglomerados, considerando-se os 11 fatores estudados.

Os fatores Competição e Financiadores apresentaram os menores escores médios, tanto no grupo de CONCORDANTES quanto no de DISCORDANTES. 
Por outro lado, em ambos os grupos, os maiores escores médios ocorreram nos fatores Confiança e Gestão (Tabela 5).

\section{Formação de Aglomerados por Segmento da Amostra}

Analisou-se, em seguida, a composição dos dois conglomerados, considerando-se os mesmos segmentos amostrais considerados no item Segmentação da amostra anterior. Para cada um dos 11 grupos constituídos, a distribuição de respondentes ficou muito próxima de $90 \%$ para os CONCORDANTES e de $10 \%$ para os DISCORDANTES, conforme se indica na Tabela 6.

\section{Tabela 6: Caracterização dos Dois Conglomerados de Respondentes (CONCORDANTES e DISCORDANTES) segundo Segmentos da Amostra}

\begin{tabular}{lc|c}
\hline \hline & \multicolumn{2}{c}{ CARACTERIZAÇÃO DOS } \\
\multicolumn{1}{c}{ SEGMENTO DA AMOSTRA } & CONGLOMERADOS \\
\cline { 2 - 3 } & 90,8 & BAIXO \\
\hline \hline Grupo I=Doutores & 90,5 & 9,2 \\
Grupo II = Mestres e Especialistas & 90,1 & 9,5 \\
Grupo III = Experiência em Pesquisa até 15 anos & 91,3 & 9,9 \\
Grupo IV = Experiência em Pesquisa de 15 anos ou mais & 88,5 & 8,7 \\
Grupo V = Vínculo com Universidade ou Escola Isolada Pública & 11,5 \\
Grupo VI = Vínculo com Instituto de Pesquisa Federal & 97,8 & 2,2 \\
Grupo VII = Vínculo com Instituto de Pesquisa Estadual & 89,2 & 10,8 \\
Grupo VIII = Classificação CNPq 1A e 2A & 90,7 & 9,3 \\
Grupo IX = Classificação CNPq 1B e 2B & 90,9 & 9,1 \\
Grupo X= Classificação CNPq 1C e 2C & 95,5 & 4,5 \\
Grupo XI = Não recebeu classificação do CNPq & 89,9 & 10,1 \\
\hline \hline
\end{tabular}

Nota - Os grupos formados foram baseados numa análise de conglomerados com os 11 fatores estudados.

\section{Análise de Conglomerados Aplicada aos Estágios Componentes do Modelo de Análise das Redes Cooperativas}

A análise de conglomerados considerou os 11 fatores do modelo, resultantes da análise fatorial, avaliando-se separadamente cada um dos três estágios mencionados (Criação de Rede, Operação de Rede, Resultados referentes à Rede), determinando então os escores médios dos fatores em relação ao grau de concordância dos respondentes com os itens formadores das dimensões de análise. As tabelas e gráficos resultantes, que não são mostrados neste trabalho, permitem constatar o seguinte. 
1. Quanto aos dois primeiros estágios (Criação e Operação), verificou-se que os resultados são muito semelhantes aos obtidos para o conjunto dos 11 fatores, sem segmentação da amostra. De fato, para estes dois primeiros estágios, independentemente considerados, também se formaram dois conglomerados: CONCORDANTES ( $88,2 \%$ e $88,0 \%$, respectivamente), e DISCORDANTES ( $11,8 \%$ e $12,0 \%$, respectivamente), percentuais muito próximos dos correspondentes aos conglomerados formados com a amostra total, conjuntamente para os três estágios seqüenciais antes citados $($ CONCORDANTES $=90,9 \%$ e DISCORDANTES $=9,1 \%)$.

2. Surgem diferenças significativas, quando se considera separadamente o terceiro estágio (Produção de Resultados), ao invés dos 11 fatores conjuntamente. Aparece agora um terceiro conglomerado, constituído pelos pesquisadores que manifestaram uma concordância mediana (aqui chamados de MODERADOS) com relação às afirmativas correspondentes aos itens geradores da dimensão Produção de resultados. Este terceiro conglomerado parece formar-se praticamente às custas do conglomerado CONCORDANTES, como se mostra abaixo no Gráfico 1.

Como é positivo o sentido das afirmativas correspondentes às questões formadoras dos fatores que compõem a dimensão Produção de Resultados, conclui-se que o surgimento do terceiro conglomerado, a partir da origem detectada, significa que, na percepção dos pesquisadores, a concordância é menor com os fatores desta dimensão (Produção de Resultados) do que, comparativamente, com os das duas outras dimensões: Criação e implantação das redes cooperativas e Operação.

\section{Gráfico 1: Distribuição dos Respondentes quanto ao Grau de Concordância com os Itens Referentes à Dimensão 'Resultados de Redes Cooperativas'}

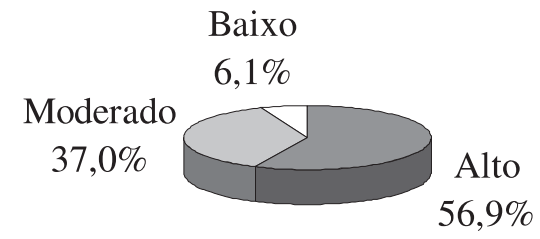

Nota: Os grupos formados foram baseados numa análise de conglomerados com os 3 fatores estudados. 


\section{Conclusões, a partir da Análise dos Resultados, na Perspectiva da Teoria Institucional}

O conjunto de dados coletados e tratados possibilita uma leitura que realça pressupostos importantes da Teoria Institucional, que serão discutidos a seguir.

\section{Mitos Racionalizados?}

A literatura sobre redes cooperativas de pesquisa enfatiza os ganhos propiciados pelos mecanismos cooperativos, não apenas em termos de eficiência, de redução de custos e prazos de execução das atividades de $\mathrm{P} \& \mathrm{D}$, mas também de maior produção científica, de benefícios sociais e de utilidade. O que se mencionou anteriormente quanto a critérios, metodologia e sistemas de avaliação das atividades de C\&T comprovou as grandes dificuldades relacionadas com a questão, reafirmando a inexistência, no Brasil, de um sistema abrangente capaz de aferir objetivamente resultados de atividades de pesquisa. Como, pois, saber se projetos executados em estruturas de redes têm maior eficiência e, principalmente, maior eficácia, se se desconhece o impacto de seus resultados? Afinal, a questão central relativa às atividades compartilhadas de pesquisa é: o mecanismo de redes efetivamente contribui para a consecução de melhores resultados? Ainda não se dispõe de dados para que, em bases científicas, se possa dar uma resposta a essa questão.

A argumentação das agências de fomento e dos órgãos de formulação de políticas em favor das ações cooperativas baseia-se fortemente na defesa da racionalidade associada à busca de maior eficiência e de aperfeiçoamento da alocação de recursos públicos. $\mathrm{O}$ argumento é tomado como verdadeiro, os pesquisadores passam a percebê-lo, aceitá-lo, legitimando-o. As agências de fomento, por sua vez, aumentam suas atividades de indução no sentido de privilegiar as ações cooperativas, propagando-as, como se demonstrou com relação à FAPEMIG (Tabela 2).

Mas seriam mesmo esses mecanismos efetivamente propiciadores de maior eficácia às atividades de pesquisa? Ou seriam mitos racionalizados, constituídos sob o argumento do aumento da eficiência e de otimização na alocação de recursos às atividades de pesquisa? Os limites da pesquisa empreendida não possibilitam uma adequada aferição de resultados, porque se baseou nas percepções manifestas dos pesquisadores com efetiva experiência em trabalhos cooperativos.

\section{Isomorfismo}

O processo de isomorfismo, no caso das organizações de $\mathrm{P} \& \mathrm{D}$, se manifesta de diversas formas, como através do esforço das entidades não apenas para 
capacitar seus quadros nas áreas que se mostram mais promissoras em cada circunstância, segundo as diretrizes das agências de fomento, mas também para continuamente ampliar e atualizar seus recursos laboratoriais, de forma a reduzir eventuais dependências em situações futuras.

Durante o tratamento dos dados coletados evidenciou-se, em diversas circunstâncias, esse fenômeno que os institucionalistas denominam isomorfismo, detectado através das percepções dos pesquisadores, como mais detalhadamente se descreveu, do ponto de vista teórico, no item Teoria Institucional.

Foram obtidas primeiramente as médias dos 11 fatores gerados pela análise fatorial, considerados os escores atribuídos por todos os respondentes, sem qualquer segmentação da amostra. As medidas descritivas das médias dos fatores foram, posteriormente, também calculadas, para fins de comparação, para segmentos da amostra determinados conforme se indicou no item Segmentação da Amostra, onde as principais conclusões são:

- não foram encontradas distinções, com significância estatística, nas percepções dos pesquisadores, quando as análises das medidas descritivas tiveram por base os segmentos definidos em função de titulação, tempo de experiência em pesquisa e nível de classificação no CNPq;

- poucas alterações surgem, todavia, quando se consideram os segmentos da amostra definidos em função da entidade a que se vincula o pesquisador, especificamente nos fatores G (Gestão) e I (Resultados não-científicos).

Os dados não deixam dúvida de que há grande proximidade, senão igualdade estatística, entre a maneira como os pesquisadores - quaisquer que sejam as categorias a que pertençam - percebem as questões consideradas no estudo, aglutinadas segundo os 11 fatores gerados a partir da análise fatorial, para analisar motivações, dinâmica e resultados relativos a trabalhos cooperativos em P\&D.

Outra forma de perceber o isomorfismo é através dos resultados da Análise de Conglomerados (Clusters Analysis), que foi empregada para identificar possíveis agrupamentos de pesquisadores da amostra, aglutinados de acordo com suas percepções sobre os 11 fatores gerados pela Análise Fatorial. Tendo sido registrada a formação de dois conglomerados, o primeiro constituído por 90,9\% dos pesquisadores (CONCORDANTES) e o segundo formado por 9,1\% dos respondentes (DISCORDANTES), a enorme desproporcionalidade comprovada entre os dois agrupamentos, além do fato de que a identificação pretendida com a análise fatorial apenas distinguiu dois grupos, são também fortes indicadores de uma tendência à uniformidade, na percepção dos pesquisadores, quanto aos fatores gerados pela técnica estatística. Além do mais, quando se considerou cada 
segmento da amostra, manteve-se o mesmo critério de subdivisão em dois grupos (CONCORDANTES e DISCORDANTES), decorrente da análise de conglomerados, em proporções não muito distantes daquelas obtidas para a amostra como um todo.

\section{Pressões Institucionais}

O tratamento dos dados coletados revela, pelo menos parcialmente, a origem, na pesquisa, de pressões coercitivas para o comportamento isomórfico. Isto não quer dizer, contudo, que não possam estar ocorrendo também pressões normativas e/ou pressões miméticas, que não emergiram de forma natural das informações obtidas. Eis alguns elementos que merecem realce.

a) A alta porcentagem $(62,05 \%)$ de redes e projetos cooperativos identificados surge como resposta a ações indutoras das agências financiadoras, por meio de editais.

b) A participação elevada das instituições governamentais no financiamento (Tabela 1), no caso das 79 redes e projetos cooperativos considerados na pesquisa: 95 ocorrências $(81,2 \%)$, enquanto empresas privadas ou entidades de classe do setor privado comparecem com 12 participações $(10,26 \%)$.

c) A instituição de programas especiais, de natureza cooperativa, no âmbito do Ministério de Ciência e Tecnologia: Programa de Apoio ao Desenvolvimento Científico e Tecnológico - PADCT, iniciada em 1991; Programa RECOPE, coordenado pela FINEP, com início em 1997.

d) A tendência crescente de a FAPEMIG trabalhar por meio de indução de atividades mediante editais, como indicam dados levantados sobre a questão.

e) O mais frequiente $(50,47 \%)$ instrumento de formalização do trabalho cooperativo é o contrato com órgão financiador, conforme comprovam dados colhidos (não reproduzidos neste trabalho), e que evidenciam a ocorrência de pressões institucionais, já que a exigência de parceria entre entidades executantes tem sido condição de habilitação incluída na maioria dos Editais das agências de fomento à Pesquisa. A justificativa geralmente apresentada nesses editais pelas agências de fomento é a de que o mecanismo de trabalho cooperativo contribui para a racionalidade da aplicação dos recursos e aumento de eficiência das ações. 


\section{O Processo de Institucionalização das Redes e Projetos Cooperativos}

Nesta perspectiva, os resultados obtidos na pesquisa e as referências extraídas da literatura possibilitam a conclusão de que o mecanismo de redes se encontra em processo de pré-institucionalização, pelas razões seguintes:

a) as agências de fomento, tanto no nível federal quanto no estadual, enfrentando problemas crônicos de falta de recursos, buscam mudanças organizacionais - adoção do mecanismo de redes - provavelmente visando atenuar suas dificuldades e incertezas;

b) a indução de atividades cooperativas, surgida no início da década de 90 nas agências federais, de forma tímida ainda na Fase II do PADCT, torna-se crescentemente mais intensa, a partir de 1996 (RECOPE, PADCT III);

c) o incentivo à formação de alianças para atividades de $\mathrm{P} \& \mathrm{D}$ propaga-se para as ações das FAP regionais;

d) As instituições promotoras das ações cooperativas, em número reduzido e razoavelmente bem articuladas entre si, favorecem os comportamentos miméticos entre as agências de financiamento;

e) as instituições promotoras são homogêneas, em termos de missão institucional, em grau elevado;

f) os esforços de teorização são praticamente inexistentes, como se verificou na literatura, para compor o referencial teórico deste trabalho;

g) Os pesquisadores executores das atividades de $\mathrm{P} \& \mathrm{D}$, sujeitos à pressão coercitiva das agências financiadoras, acabam por conferir consenso social e legitimidade para estas estruturas, como o confirmam os dados coletados e tratados nesta pesquisa.

\section{ComentÁrios Finais}

A Teoria Institucional demonstrou ser ferramenta potente para explicar fatos relacionados com a percepção dos pesquisadores quanto a aspectos relativos às suas motivações para o trabalho cooperativo, à estabilidade das parcerias de $\mathrm{P} \& \mathrm{D}$ constituídas e à efetividade do mecanismo de redes, para assegurar melhores resultados para os trabalhos de pesquisa. A pesquisa, que constitui 
uma pouco comum aplicação empírica da Teoria Institucional, revelou posições e comportamentos fortemente isomórficos e possibilitou identificar potenciais fontes de pressões coercitivas para o trabalho cooperativo em C\&T. Esclareceu ainda que mecanismo de parcerias para execução de atividades de P\&D, no Brasil, ainda não se encontra em fase de plena institucionalização, carecendo de novos estudos que sejam capazes de demonstrar efetivamente que o mecanismo de redes é capaz de propiciar a consecução de melhores resultados para as atividades de pesquisa, em termos quantitativos e qualitativos.

\section{Artigo recebido em 04.11.2004. Aprovado em 05.04.2005.}

\section{NOTAS}

${ }^{1}$ Fundação de Amparo à Pesquisa do Estado de Minas Gerais - FAPEMIG.

${ }^{2} \mathrm{O}$ número de conexões possíveis entre $\mathrm{n}$ pontos é dado pela fórmula: $\mathrm{nC} 2=\mathrm{n} ! /(\mathrm{n}-2) ! 2$ ! . Assim, o número de ligações possíveis entre 6 indivíduos é 15 . Se acrescentarmos 1 indivíduo (n=7), o número de interações possíveis muda para 21.

${ }^{3}$ Fonte: Empresa Brasileira de Pesquisa Agropecuária - EMBRAPA (2001). Plano de ação para 2002 [Folheto]. Sete Lagoas: CNPMS/EMBRAPA.

${ }^{4}$ Vasconcelos, F., \& Machado-da-Silva, C. (Org). (2003). Fórum Especial: A teoria institucional em um contexto brasileiro: dinâmicas de inovação e imitação (Chamada de Trabalhos). Revista de Administração de Empresas, 43(1), p. 7.

${ }^{5}$ Artigo original publicado em 1977 no American Journal of Sociology, 83, 340-63. Institutionalized organizations: formal structure as myth as ceremony. In W. W. PowelL \& P. J. DiMaggio. The New Institutionalism in Organizational Analysis. Chicago: The University of Chicago Press. 1991 (Reedição).

${ }^{6}$ Artigo original publicado em 1983 na American Sociological Review, 48, 137-60.

${ }^{7}$ Idem, nota anterior .

${ }^{8}$ Artigo reeditado em 1991 (Ver Nota 5).

${ }^{9}$ Há autores que desdobram o construto eficácia nos construtos eficiência, resultados científicos, benefícios sociais e utilidade.

${ }^{10}$ Artigo original, com o mesmo título, publicado em 1977, no American Journal of Sociology. 83, 340-63.

${ }^{11}$ Banco de Dados phpESP (software livre disponível na web).

${ }^{12}$ Diretório dos Grupos de Pesquisa do CNPq - 2002.

${ }^{13} \mathrm{p}=$ Nível de Significância. Teste $\mathrm{t}$ de Student para amostras independentes. No caso, a hipótese nula é para Grupo I = Grupo II: isto então só não se confirmará se p<0,05. ( $95 \%$ de confiança). 


\section{ReferênCias Bibliográficas}

Assunção, F. C. R. (n.d.).

A pesquisa cooperativa como solução para o desenvolvimento tecnológico. Recuperado em 11 julho, 2001, de http:/ /www.engenheiro2001.org.br/ programas/980215a2.htm

Burns, T., \&

Stalker, G. M. (1961).

The management of innovation. London: Tavistock.

Casson, M., \&

Cox, H. (1999).

An economic model of inter-firm networks. In M. Ebers (Ed.). The formation of inter-organizational networks (pp. 174-196). Oxford: Oxford University Press.

Castells, M. (1999).

A sociedade em rede (Vol. 1, Cap. 3, pp. 173-218). Rio de Janeiro: Paz e Terra.

Chotangada, R. (1997).

A governance systems that facilitate innovation: changing perspectives of supplier customer relationships. the case of the automotive industry. Tese de doutorado, University of Cincinnati, Cincinnati, Ohio, USA.

De Laat, P. (1999).

Research and development alliances: ensuring trust by mutual commitments. In M. Ebers (Ed.). The formation of inter-organizational networks (pp. 146-173). Oxford: Oxford University Press.
DiMaggio, P. J., \&

Powell, W. W. (1991).

The iron cage revisited: institutional isomorphism and collective rationality in organizational fields. In W. W. Powel \& P. J. DiMaggio (Eds.). The new institutionalism in organizational analysis. Chicago, Il: The University of Chicago Press.

Hatch, M. J. (1997).

Organization theory: modern, symbolic and postmodern perspectives (p. 299). New York: Oxford University Press.

Longo, W. P., \&

Oliveira, A. R. P. (1999).

Pesquisa cooperativa e centros de excelência. Recuperado em 17 julho, 2001, de http://www.mct.gov.br/cee/ revista/parcerias9/13revista

Longo, W. P., \&

Weisz, J. (2000).

Technological innovation networking in Brazil: an assessment of the RECOPE sub-program. Proceedings of the Triple Helix International Conference, Rio de Janeiro, RJ, Brasil, 3.

Loughlin, D. M., \&

Búrca, S. (1995).

The grounded theory alternative in business network research [DCUBS Research Papers No 4], Dublin City University, Dublin 9, Ireland. 
Lütz, S. (1999).

Learning through intermediaries: the case of inter-firm research collaborations. In M. Ebers (Ed.). The formation of inter-organizational networks (pp. 146-173). Oxford: Oxford University Press.

Machado-da-Silva, C. L., \&

Gonçalves, S. A. (1999).

A teoria institucional. (Nota Técnica). In S. Clegg, C. Hardy, \& W. Nord (Eds.). Handbook de estudos organizacionais (pp. 200-226). São Paulo: Atlas.

Malhotra, N. K. (2001).

Pesquisa de marketing: uma orientação aplicada (3a ed.). Porto Alegre: Bookman.

March, J. G., \&

Olsen, J. P. (1989).

Rediscovering institutions: the organizational basis of politics. New York: The Free Press.

Mazzali, L., \&

Costa, V. M. H. M. (1997).

As formas de organização em 'em rede': configuração e instrumento de análise da dinâmica industrial recente. Revista de Economia Política, 17(4), 121- 139.

Meyer, J. W., \&

Rowan, B. (1991).

Institutionalized organizations: formal structure as myth as ceremony. In W. W. Powell \& P. J. DiMaggio (Eds.). The new institutionalism in organizational analysis. Chicago: The University of Chicago Press.
Nelson, R. (1993).

National innovation systems: a comparative analysis. Oxford: Oxford University.

Nohria, N. (1992).

Is a network perspective a useful way of studying organizations? In $\mathrm{N}$. Nohria \& R. G. Eccles (Eds.). Networks and organizations: structure, form, and action (pp. 1-22). Boston: Harvard Business School Press.

Ouchi, W. G., \&

Bolton, M. K. (1988).

The logic of joint research and development. California Management Review, 30(3), 9-33.

Porter, M. (1999).

Competição: on competition: estratégias competitivas essenciais (6a ed., p. 515). Rio de Janeiro: Campus.

Powell, W. W. (1991).

Neither market nor hierarchy: network forms of organization. In $\mathrm{G}$. Thompson, J. Frances, R. Levacic, \& J. C. Mitchell (Eds.). Markets, hierarchies and networks: coordination of social life (pp. 259276). London: Sage.

Powell, W. W., \&

DiMaggio, P. J. (1991).

The new institutionalism in organizational analysis. Chicago, Il: The University of Chicago Press.

Ring, P. S. (1999).

Processes facilitating reliance on trust in inter-organization networks. In M. Ebers (Ed.). The formation of interorganizational networks (pp. 113145). Oxford: Oxford University Press. 
Sampieri, R. H.,

Collado, C. F., \&

Lucio, P. B. (2003).

Metodología de la investigación (3a ed., p. 704). México: McGrawHill.

Schibany, A., \&

Polt, W. (2001). Innovation and networks: an introduction to the theme. In OCDE (Ed.). Innovative networks. cooperation in national innovation systems (pp. 7-13). Paris: Author.

Scott, R.W. (1995).

Introduction: institutional theory and organizations. In R. W. Scott \& S. Christensen (Eds.). The institutional construction of organizations: international and longitudinal studies (pp. xi-xxiii). London: Sage

Sebastián, J. (1999).

Analisis de las redes de investigacion de America Latina con la Unión Europea. RECITEC - Revista de Ciência e Tecnologia, 3(2), 308-321.
Selznick, P. (1948).

Foundations of the theory of organization. American Sociological Review, 13(1), 25-35.

Simon, H. (1978).

Rationality as process and as product of thought. American Economic Review, 68(2), 1-16.

Tolbert, P. S., \& Zucker, L. G. (1999).

A institucionalização da teoria institucional. In S. Clegg, C. Hardy, \& W. Nord (Eds.). Handbook de estudos organizacionais (pp. 220-226). São Paulo: Atlas.

Weisz, J., \&

Roco, M. C. (1995).

Engineering research and education networking in the Americas. In OTA (Ed.). International partnership in large science projects (Appendix pp. 96-114). (OTA-BP-ETI-150. Govern. P. Office).Washington, DC: Congress Office of Technology Assessment. 